\title{
Féminité et marginalité dans la prose postcoloniale : essai de réflexion sur la polygamie et le mariage arrangé dans Celles qui attendent de Fatou Diome
}

\author{
Arsène Magnima Kakassa \\ Université Omar Bongo/Libreville \\ arsene.magnima@yahoo.fr
}

Rebut: 29 d'abril de 2018

Acceptat: 7 de juliol de 2018

\section{RESUM}

Feminitat i marginalitat a la prosa postcolonial: assaig de reflexió sobre la poligàmia i el matrimoni arreglat a Celles qui attendent de Fatou Diome Aquesta reflexió argumenta que la literatura africana sempre ha presentat personatges femenins marginats i dominats pels homes. Diome, com escriptores com Mariama Bâ (Une si longue lettre, 2001), Maryse Conde (Moi Tituba sorcière, 1986), etc., representa figures femenines amb destins complexos, exclosos de tots els òrgans de decisió de la vida social i que estan experimentant la poligàmia i el matrimoni concertat, malauradament, legitimats per les seves respectives societats. Aquest article postula que l'espai africà en general i el de Celles qui attendent (2010) tenen en comú la imatge de les dones confinades pel seu sexe en rols opressius reforçats per les institucions patriarcals. En altres paraules, aquesta novel.la revela un costat de la tradició africana que ha quedat obsolet, i les pràctiques que han de ser desafiades per trencar finalment els estereotips i la manipulació orquestrada pels homes.

PARAUles ClAU

Estereotips, matrimoni arreglat, poligàmia, tradició africana, dona. 
RÉSUMÉ

Féminité et marginalité dans la prose postcoloniale : essai de réflexion sur la polygamie et le mariage arrangé dans Celles qui attendent de Fatou Diome

Cette réflexion soutient que la littérature africaine a toujours présenté des personnages féminins marginalisés et dominés par les hommes. Diome, à l'instar des écrivains comme Mariama Bâ (Une si longue lettre 2001), Maryse Conde (Moi Tituba sorcière 1986), etc., représente des figures féminines aux destins complexes, exclues de toutes instances décisionnelles de la sociale, et subissant la polygamie et le mariage arrangé, malheureusement, légitimés par leurs sociétés respectives. Cet article postule que l'espace africain en général et celui de Celles qui attendent (2010), ont en commun l'image de femmes confinées par leur sexe dans des rôles opprimants renforcés par les institutions patriarcales. Autrement dit, ce roman dévoile un pan de la tradition africaine devenue caduque, et dont il faut récuser les pratiques pour sortir enfin des stéréotypes et de la manipulation orchestrés par les hommes.

MoTs CLÉS

Stéréotypes, mariage arrangé, polygamie, tradition africaine, femmes.

\section{RESUMEN}

Feminidad y marginalidad en la prosa postcolonial: ensayo de reflexión sobre la poligamia y el matrimonio concertado en Celles qui attendent de Fatou Diome

Esta reflexión sostiene que la literatura africana siempre ha presentado personajes femeninos marginados y dominados por hombres. Diome, como escritoras como Mariama Bâ (Une si longue lettre 2001), Maryse Conde (Moi Tituba sorcière 1986), etc., representa figuras femeninas con destinos complejos, excluidas de todas las instancias de toma de decisiones de lo social, y sometidas a la poligamia y al matrimonio arreglado, desafortunadamente, legitimados por sus respectivas sociedades. Este artículo postula que el espacio africano en general y el de Celles qui attendent (2010) tienen en común la imagen de mujeres confinadas por su sexo en roles opresivos reforzados por las instituciones patriarcales. En otras palabras, esta novela revela un lado de la tradición africana que se ha vuelto obsoleta, y cuyas prácticas deben desafiarse para finalmente romper con los estereotipos y la manipulación orquestada por los hombres.

Palabras Clave

Estereotipos, matrimonio convenido, poligamia, tradición africana, mujer. 


\begin{abstract}
Femininity and marginality in postcolonial prose : a reflection on polygamy and marriage arranged in Celles qui attendent for Fatou Diome

Our paper holds that African Literature has always presented female characters as being marginalized and dominated by male. Diome, like other writers as Mariama Bâ (Une si longue lettre, 2001), Maryse Conde (Moi Tituba sorcière, 1986) etc, portrays female figures whith complicated future, excluded from every decision-making body. They are subject to polygamy and arranged marriage, unfortunately legitimated in the society they belong to. This paper demonstrates that the African space in general and that of Celles qui attendent (2010) share the image of women confined in oppressive roles because of their gender, situation that is reinforced by patriarchal institutions. In other words, this novels unveils a side of African tradition now obsolete, which has to disappear in order to end stereotypes and men manipulations.
\end{abstract}

KEYWORDS

Stereotypes, Arranged marriage, Polygamy, African tradition, Women.

\title{
Introduction
}

La polygamie et le mariage arrangé sont autorisés et pratiqués à la fois dans tous les pays de l'Afrique de Nord de culture arabo-musulmane (sauf la Tunisie) et en Afrique Sub-saharienne de croyance animiste ou de culture musulmane ${ }^{1}$. Pour les Africains, la polygamie est une question d'héritage culturel. Cependant, cette pratique sociale ne cadre plus avec les exigences du monde actuel, car elle ne facilite pas l'épanouissement des figures féminines. En effet, depuis les siècles précédents, le récit sur les femmes est constitué de violences, de frustrations et de privations des droits les plus élémentaires, comme le droit de disposer de son corps, d'aimer et de choisir librement son conjoint. De nombreuses contrées africaines demeurent encore assujetties aux pratiques traditionnelles d'un autre âge - notamment l'excision, la polygamie, le mariage foré ou arrangé - qui enferment les femmes dans le silence. De

\footnotetext{
${ }^{1}$ Voir : Kebme MILOLO, L'image de la femme chez les romancières de l'Afrique Noire francophone, Éditions universitaires, Fribourg Suisse, 1985.
} 
nombreux romans africains évoquaient ces faits sans véritablement les dénoncer. Batouala ${ }^{2}$ de René Maran par exemple, s'intéresse à l'histoire d'un chef de tribu africain dénommé Batouala. Un polygame qui a neuf épouses. En plein temps colonial, les critiques de l'auteur sont plus portées vers le système colonial que sur la polygamie qui est considérée comme une pratique banale. Les Soleils des indépendances ${ }^{3}$, de son côté, aborde des thèmes en rapport avec la condition de la femme sans émettre une critique réelle. Kourouma parle de l'excision, la polygamie, le lévirat et de la sorcellerie qui est à l'origine de la stérilité de Salimata la première femme de Fama.

Cependant, depuis les années 70, les intellectuels de tout bord, les artistes et les écrivains commencent à critiquer ces pratiques traditionnelles qu'ils trouvent rétrogrades. On peut notamment citer, Une si longue lettre 4 de Mariama Bâ, considéré comme l'un des premiers véritables romans écrits par une africaine, et $Z a l a^{5}$ de Sembène Ousmane, qui dénoncent violemment les pratiques de la polygamie dans les sociétés ouest-africaines. CoqueryVidrovitch ${ }^{6}$, justifie cet éveil de conscience en se référant aux grandes mutations mondiales au niveau politique, économique et social, dont les chocs successifs sont éprouvés par le continent noir, qui ont leurs impacts sur la société et la femme africaine. La colonisation, les deux guerres mondiales, les processus de la décolonisation, l'acquisition de l'indépendance et enfin la longue dépression qui règne sur les pays africains après l'indépendance et qui remonte au début des années 1970.

Cette réflexion s'articule également autour de la notion de la marginalité 7 , dans notre perspective, tout ce qui ramène à une relative liberté

${ }^{2}$ René MARAN, Batouala, Éditons Allbin Michel S.A, Paris, 1938.

${ }^{3}$ Ahmadou KOUROUMA, Les Soleils des Indépendances, Éditions du Seuil, Paris, 1968.

${ }^{4}$ Mariama BÂ, Une si longue lettre, Serpent à plumes, Paris, 2001.

${ }^{5}$ Sembene OUSMANE, Xala, Présence Africaine, Paris, 1973.

${ }^{6}$ Catherine COQUERY-VIDROVITCH est historienne et spécialiste reconnue de l'histoire de l'Afrique noire. Dans son ouvrage, Les Africaines. Histoire des femmes d'Afrique noire du XIX ${ }^{e}$ au XX $X^{e}$ siècle (Paris, éditions Desjonquères 1994), elle se penche sur le destin mouvementé des femmes africaines en étudiant leur rôle et leur fonction dans leurs sociétés respectives. Pour elle, la plupart de ces femmes sont mises au ban, exploitées et « dressées » à la passivité et à l'humiliation. Aussi, elles sont certes valorisées comme productrices et reproductrices mais elles restent un bien à exploiter, puisqu' ayant une valeur sociale et marchand.

${ }^{7}$ Comprendre marginalité dans le sens d'être « en marge de ». Un pan de la critique féministe nous montre comment les femmes sont renvoyées à une double marginalité. La première, implicite, transversale à toute pratique, tout discours : être femme c'est ne pas être homme, c'est vivre à partir de, en marge d'un homme, et ce sont les discours juridiques qui le manifestent le plus explicitement ; la seconde, être femme, c'est être Mère et c'est dire que, dans une vie de femme, 
ou autonomie. C'est-à-dire ce qui renvoie aux minorités, à ceux qui n'ont pas la parole ou de représentation dans une société quelconque. Tout ce qui réside sous les injonctions d'une autorité institutionnelle ou sociale. Dans notre cas, la marginalité est assignée aux personnages féminins qui évoluent sous l'autorité parentale ou sous l'influence des traditions. En accord avec notre propos, la marginalité a donc un lien avec les questions de genre et de sexe.

Dans Celles qui attendent ${ }^{8}$, Fatou Diome critique et montre comment les femmes sont victimes de cette marginalité ambiante et comment elles restent inféodées aux hommes. Ce sont pour la plupart des êtres vivant « aux marges de l'histoire », exclus de toute dynamique sociale, privés d'identité et réduits à la subordination par les groupes sociaux hégémoniques et dominants ${ }^{9}$. Aujourd'hui, de nombreux écrivains africains optent pour des héroïnes qui sortent des sentiers battus et préfèrent aller contre un destin tout tracé. Leur rébellion se manifeste ainsi sous plusieurs formes : contre l'ordre patriarcal et familial, contre les attentes et les fantasmes des sociétés masculines et coloniales. Le prix à payer pour une telle confrontation des valeurs et des idéaux socialement établis s'avère souvent très lourd pour les personnages féminins. Diome examine la position féminine au sein d'un corps social fortement hiérarchisé, duquel certaines héroïnes tentent de se détacher.

Nous nous servons de la notion de subalterne que Spivak emprunte à Gramsi, et qu'il ne faut pas confondre avec les concepts de dominé ou d'opprimé. Elle renvoie en fait à la position des femmes du Tiers-Monde que Spivak considérerait comme des sans voix, exclues de la sphère du discours et de la représentation. La position subalterne des femmes, notamment, celles des femmes africaines doit être analysée en tenant compte des considérations sociohistoriques, anthropologiques, et culturelles. C'est pourquoi, les réflexions de Coquery-Vidrovitch et de Simone de Beauvoir, peuvent également être utiles pour comprendre certains aspects de la condition des femmes. Cet article a pour objectif de voir comment la polygamie et le mariage arrangé enferment, dans Celles qui attendent, les femmes dans une condition insatisfaisante et sans issue.

tout aspect autre que ce rapport totalitaire à la maternité ne peut se vivre qu'en marge de celle-ci, à son extrême périphérie. $C f$. Christiane BERNIER, « Normes et marginalités. Comportements féminins aux $19^{\mathrm{e}}$ et $20^{\mathrm{e}}$ siècles », Recherches féministes, 1992, n5(1), pp. 186-189, p. 186-187.

${ }^{8}$ Fatou Diome, Celles qui attendent, Éditions Flammarion, Paris, 2010. Pour citer le texte de Diome, nous avons retenu l'abréviation suivante : $C Q A$.

${ }^{9}$ Gayatri Spivak, Les Subalternes peuvent-elles parler ? Traduit de l'anglais par Jérôme Vidal. Éditions Amsterdam, Paris, 2006, p. 49. 


\section{La polygamie et significations}

\subsection{Mal être et frustration}

Sous le sous-titre « Pourquoi la polygamie ? » Bestman fait une étude intéressante de cette pratique matrimoniale en Afrique. Il soutient qu'au sein de la société traditionnelle africaine, la polygamie peut être expliquée à partir de la métaphysique africaine, par le problème de l'immortalité. Ceci est lié au culte des Ancêtres, selon lequel ils continuent à vivre parmi leur descendance, et disparaitront seulement quand leur lignée sera coupée par le manque de descendants. Ce «prolongement cosmique » ne peut être garanti que par la naissance de beaucoup d'enfants et, pour cette raison, une épouse stérile est déclarée inutile puisque la femme symbolise la fécondité même ${ }^{10}$. Ici, le mariage polygame s'explique par des raisons de pérennité du clan, de la famille ou de la communauté. La polygamie a énergiquement résisté aux missionnaires, aux colonisateurs, puis aux législations des États africains indépendants. Cette stabilité est celle des structures anthropologiques dont font partie les systèmes matrimoniaux ${ }^{11}$. En d'autres termes, cette forme de mariage est ancrée dans les us et coutumes, dans l'imaginaire africain, ce qui explique certainement sa résistance face à tous les systèmes politiques et sociaux qui ont traversé ce continent.

Avant Diome, deux écrivains sénégalais cités ci-dessus s'étaient déjà saisi de la condition de la femme africaine, en dénonçant la polygamie et le mariage arrangé. Dans Celles qui attendent, la religion musulmane est le bras séculier de ces pratiques matrimoniales ${ }^{12}$, puisqu'elles assurent selon elle, la survie du clan familial. Puisque ce sont les femmes qui sont chargées de travaux agricoles, " plus les épouses sont nombreuses, plus grande est la superficie qui peut être cultivée $»{ }^{13}$ En revanche, il est indéniable qu'elle

\footnotetext{
${ }^{10}$ Martin T. BESTMAN, Sembene Ousmane et l'esthétique du roman négro-africain, Québec, Naaman, 1981, p. 70.

${ }^{11}$ Voir : Dominique TABUTIN, «Les populations de l'Afrique au Sud du Sahara », Population et société, 1989, n²31, pp. 40-50.

${ }^{12}$ Ce postulat est remis en cause par Kebme Milolo. Pour lui, la polygamie est une institution spécifiquement africaine héritée de la tradition. Ce n'est pas une conséquence de l'introduction de l'Islam sur ce continent. Ceci dit en analysant ce phénomène, il faut étudier le mariage polygamique et la famille polygamique pour eux-mêmes, tel qu'ils sont et non plus seulement en rapport avec la civilisation musulmane. Voir son ouvrage : L'image de la femme chez les romancières de l'Afrique Noire francophone, (Editions universitaires, Fribourg Suisse, 1985).

${ }^{13}$ Achola O PALA et Madina LY, La femme africaine dans la société précoloniale, Unesco, Paris, 1979, p. 52.
} 
s'opère dans une suprématie du masculin qui confine les femmes au rang de coépouses souvent insatisfaites, enclines à d'incessantes jalousies et rivalités. Selon les considérations socio-historiques et culturelles, la polygamie confère un prestige social dans de nombreuses contrées africaines.

Le roman Celles qui attendent pose deux personnages principaux en position polygamique : Bougna et Coumba, bien que de générations différentes, vivent toutes deux les frustrations de la polygamie. Elles y opposent cependant des attitudes distinctes. Bougna en a fait le combat de toute une vie : «Bougna était de ces femmes qui font de la polygamie un conflit permanent. Depuis son mariage [avec Wagane], la concurrence et la rivalité l'occupaient du matin au soir » (CQA, p. 49).

Afin de se soustraire de son statut de seconde épouse, ses gestes sont manigancés dans le but d'outrepasser le pouvoir de la première. C'est pour cette raison qu'Irène Assiba d'Almeida note que dans un contexte polygamique, la multiplication des femmes équivaut à leur négation, et favorise une véritable compétition interne ${ }^{14}$. Dans le cas de Bougna, le texte fournit ses formulations de défense contre son état féminin jugé dégradant : elle est tour à tour « retorse et provocante envers ses coépouses » (CQA, p. 48), traite sa rivale de "pimbêche » $(C Q A$, p. 38), complote sans fin une vengeance sournoise ( $C Q A$, p. 24) et, elle traite son mari d'« injuste » (CQA, p. 38) et responsable de cette situation conjugale. Disgrâce, sentiment d'abandon, rancune tenace, sont les maîtres mots qui structurent la vie de Bougna dans le récit. D'ailleurs, un jour, "se jurait-elle, elle laverait l'affront » $(C Q A$, p. 52) ; l'affront de ne pas être la seule maîtresse de la demeure et des sentiments de Wagane ; affront, pour Bougna, de savoir ses enfants moins scolarisés. C'est d'ailleurs dans cette optique, qu'en vue d'acquérir un statut social plus enviable auprès de sa coépouse, que Bougna convint Arame d'envoyer clandestinement leurs fils à l'étranger. Selon ses espérances, l'exil de leurs fils pourra les revaloriser et améliorer leurs conditions de vie.

L'état de Bougna n'est appelé à aucune amélioration tangible au cours de la trame romanesque. Bien qu'elle clame son insatisfaction dans son foyer conjugal, elle s'y conforme néanmoins : elle demeure avec Wagane malgré les piètres conditions de vie, malgré la décision de ce dernier de prendre une troisième épouse. « [...] Elle n'entendait pas céder un pouce de la place qui était la sienne. Trop fière pour prendre la fuite, elle se convainquit qu'elle

${ }^{14}$ Irène ASSIBA D'ALMEIDA et Sion HAMOU, " L'écriture féminine en Afrique noire francophone », Études littéraires, Québec, 1991, vol. 24, n², p. 44. 
devait rester et lutter pied à pied jusqu' au jour où elle récolterait les lauriers de son combat» (CQA, p. 52).

La deuxième figure féminine à subir ce contexte polygame dénoncé par le roman est Coumba. Épouse d'Issa, le fils de Bougna, elle se voit imposer ce système lorsqu'il revient d'exil au bras d'une deuxième femme, européenne, prise en son absence. N'ayant ni su ni souhaité cette union, Coumba, épuisée par le désespoir engendré par l'attente, n'y oppose aucune résistance et se plie aux règles établies par sa nouvelle coépouse. Issa sera avec Coumba à Niodior un mois par année, et repartira ensuite auprès de sa deuxième famille en Europe. Ici, l'épouse africaine légitime et approuvée par l'entourage d'Issa est outrepassée par le pouvoir de l'argent de la seconde épouse, dont « seul son argent la rendait supportable au sein de cette famille dans le besoin » (CQA, p. 269). Pour les habitants du village, l'attrait de ces moyens financiers les pousse même à valoriser cette union polygamique : «Au lieu de [reprocher à Issa] son immense trahison, on le regardait, on le scrutait, on l'admirait, comme on se laisse ébahir par ceux qui ont marché sur la Lune » (CQA, p. 268).

Même si les secondes noces d'Issa se révèlent fortement désavantageuses pour Coumba. Elle est tout de même parrainée monétairement par l'autre famille de son mari, ce qui l'aide à prendre soin des deux enfants nés de son mariage avec Issa. En addition à cela, cet arrangement lui est indéniablement défavorable en ce qui concerne ses besoins affectifs, car elle demeure séparée de son mari par une distance géographique et culturelle qui la prive de tous contacts physiques avec son époux quasiment toute l'année. Résignée à ce sort malheureux, Coumba s'y soumet sans céder à la possibilité de briser son union, puisque l'ampleur de la pression sociale l'en empêche : "Coumba se disait qu'elle ferait mieux de reprendre sa liberté. Mais elle savait qu'au village elle aurait eu toutes les peines du monde à faire valoir un tel choix» (CQA, p. 220). C'est pourquoi pour Beauvoir, il n'y a pas de « symétrie dans la situation du couple $»^{15}$, la femme se heurte à un phénomène culture : l'oppression de l'homme sur la femme.

Le contexte socio-anthropologique ne lui permet pas d'émettre une quelconque idée de divorce. Son mariage avec Issa engage, le clan, la famille, en un mot, la communauté. Coumba est alors dans une condition d'insatisfaction totale : cela se traduit par une profonde dégradation personnelle. Cette détérioration suscite le respect et l'admiration de sa communauté puisqu'elle est une conséquence directe de la fidélité applicable par l'épouse en circonstance

\footnotetext{
${ }^{15}$ Simone de Beauvoir, Le Deuxième sexe, Tome II, Éditions Gallimard, Coll. « Idées », SaintAmand, 1949, p. 197.
} 
d'exil. Sa position de martyre occasionnée par l'attente applique le sceau de cette loyauté à la mémoire d'Issa dont elle se languit et au corps social qu'elle intègre sans transgressions. Son fils, reconnu parce que légitimement conçu avec son mari, grandit au milieu d'une cohésion sociale tissée de tendresse comme de sympathie pour son statut d'orphelin de père. Chez Diome, la polygamie assure le prestige le social et le prolongement du clan, de la famille. Elle ne se pratique pas forcément à cause de la stérilité des femmes.

\subsection{Acceptation et résignation}

La passivité de Bougna et de Coumba traduit un désir inassouvi de monogamie. Une société à forte tendance polygame les confine, en tant que sujets féminins, à des ménages polygyniques ${ }^{16}$ inadaptés à leur idée du bonheur conjugal. Bougna, la première épouse de Wagane, «moulée dans les certitudes traditionnelles, considérait la polygamie comme une situation inévitable [...] Elle n'était pas indifférente, loin de là, mais elle savait qu' aucune de ses colères ne changerait la couleur du ciel. Elle voulait simplement rester digne, tenir, ne pas perdre la face devant la nouvelle venue » $(C Q A$, p. 49). Elle a naguère incarné le rôle de la fraîche et jeune nouvelle venue qui, une fois insérée dans un mariage déjà établi, capte en premier lieu toute l'attention du mari. Coumba, quant à elle, entre dans son union avec Issa munie d'une mentalité monogame tissée par les images d'un couple fort, uni et clos. «En épousant Issa, elle avait rêvé d'amour, de douceur, de complicité, de nuits torrides, mais certainement pas de cette solitude qui s'apparentait à un interminable veuvage » $(C Q A$, p. 211). Après de nombreuses années à vivre dans les affres de l'attente du retour d'Issa (émigré en France) et à se languir de lui dans sa « cage virtuelle» $(C Q A$, p. 215), Coumba réalise que sa vision idéaliste de la vie de femme mariée n'aura été que chimère. C'est pour cette raison que Simone de BEAUVOIR, citée par Rueschmann, affirme que «le couple marié [est] une entité hypocrite $»^{17}$, et une « maison-prison pour les femmes $»^{18}$.

À ce désenchantement s'ajoute le fait que le mariage ne sert pas seulement les intérêts des principaux acteurs, mais aussi ceux du groupe social. Pour les habitants de Niodior, le couple marié unit deux clans et englobe, à

\footnotetext{
${ }^{16}$ Spécificité d'une union polygamique, où l'homme a plusieurs épouses ou partenaires (au contraire d'une union polyandrique, laquelle supposerait une femme ayant plusieurs époux ou partenaires).

${ }^{17}$ Anne E. BROWN and Marjanne E. GOOZE (Editors), International Women's Writing New Landscapes of Identity, Greenwood Press, Westport, Connecticut, 1995, p. 12.

${ }^{18}$ Anne E. BROWN and Marjanne E. GOOZE, op.cit., p. 11.
} 
une échelle plus large, toute la société. « [Tout] le monde l'avait exhortée à se montrer à la hauteur de ce qu'on attendait d'elle, comme si [Coumba] ellemême n'attendait rien de son mariage » (CQA, p. 163). La pression du groupe, dans ce cas, réduit les marges manœuvre de Coumba et limite son bonheur personnel qui est désormais étroitement lié à celui de sa famille et à celui des proches d'Issa. Coumba emménage avec Bougna selon la tradition voulant que la jeune épouse intègre les quartiers de sa belle-famille pour apporter son aide à sa belle-mère dans les tâches ménagères de la maison. La jeune fille comprend que peu d'améliorations de son existence se profilent dans les nombreuses années de solitude qui l'attendent. "Une épouse doit être docile », lui avaient conseillé toutes ses aînées présentes lors de ses noces » (CQA, p. 163). Ici, le mariage a donc une valeur socio-anthropologique, puisqu'il n'engage pas seulement les individus isolés mais les groupes et permet la solidarité clanique $^{19}$.

Le mariage d'Issa avec une Française durant ses années d'exil modifie l'état conjugal de Coumba, la circonscrivant dans une relation polygame. Cet état est d'autant plus dramatique pour ce personnage qu'il lui est annoncé comme un fait accompli au retour d'Issa à Niodior, après plusieurs années d'absence. L'Européenne, consciente des avantages que lui confère son pouvoir financier, accepte de jouer « le jeu » de la polygamie auprès de Coumba. Cette dernière, réduite au rôle de la première épouse désabusée, est simple spectatrice devant ce tournant inattendu que prend son union ${ }^{20}$.

L'interminable attente pour le retour d'Issa à Niodior a considérablement affaibli Coumba, qui en subit une dévastation morale et physique : «Sa routine, c'était languir, encore et toujours » $(C Q A, 213)$. Ce désarroi l'empêche d'être animée de la même rage de combat que Bougna devant sa rivale. Le regard que porte Coumba sur son état de femme polygame est, au-delà du choc initial, résolument passif. Coumba cède tour à tour à la pression sociale et au fait accompli du mariage d'Issa à l'Européenne (duquel sont nés trois enfants métis) durant son séjour à l'étranger. Ayant à cœur le bien-être et la stabilité de son propre fils, elle entre dans une résignation complète de ses idéaux de bonheur conjugal monogamique et s'en remet en toute conformité au chemin tracé pour elle.

\footnotetext{
${ }^{19}$ Cheik Aliou NDAO, Excellence, vos épouses ! Éditions NEA, Dakar, 1993, p. 104.

${ }^{20} \mathrm{Ce}$ revers romanesque n'est pas sans rappeler la situation similaire du personnage de Ramatoulaye dans Une si longue lettre de Mariama Bâ. Incontournable figure féminine de la littérature africaine, le personnage du roman de Bâ accuse également une dégradation de son état attribuable à une polygamie imposée.
} 
Bougna et Coumba subissent les exigences de la polygamie et s'y résolvent, les pressions sociales les contraignent à l'acceptation, voire à la passivité, devant cette tradition. De ce qui précèdent, on tenant des on remarque que dans la vie réelle, les femmes africaines avaient été prises au piège dans les ramifications politiques, économiques et, bien plus important, psychologiques créées par la colonisation ${ }^{21}$ et, bien qu'elles eussent aide leurs pays à se libérer du joug du colonialisme, en combattant autant que leurs hommes, elles furent, à nouveau, après les indépendances, tôt renfermées dans la vieille tradition patriarcale.

\section{Le mariage arrangé}

Le mariage arrangé ou forcé suppose que la femme n'a pas le choix de son conjoint. Pour Chevrier, il s'agit d'un contrat social entre familles, où l'homme est la seule personne à qui on demande un avis. "La fille est priée d'obéir. Si elle refuse, maudite ou souvent on la force. Il faut respecter l'ordre établi $\gg^{22}$. Le mariage arrangé est le second fait négatif du quotidien de la femme dans Celles qui attendent. Cette pratique occupe une place prééminente dans le texte. C'est avec le personnage d'Arame que cette pratique est d'abord introduite. « $[\grave{A}]$ quarante-neuf ans, [elle] maudissait encore ses propres parents » $(C Q A$, p. 15) de leurs manigances pour l'avoir marié à Koromâk dont elle ressentait aucun sentiment. Son destin de femme s'est trouvé scellé par leur décision :

Elle avait à peine atteint sa dix-huitième année, lorsque, sans la consulter, on accorda sa main à Koromâk, un monsieur du même âge que son père. Depuis, supporter ce mariage fut son héroïsme du quotidien. Maintenant que Koromâk, vieux et malade, était devenu son fardeau, elle découvrait un autre supplice : l'obligation de prendre soin d'un être qu'elle avait toujours détesté (CQA, pp. 15-16).

Arame était respectueuse des traditions et des figures d'autorité que représentaient ses parents et sa société : « Koromâk, cet homme grognon, qu'elle subissait depuis des années, on le lui avait imposé à coups de gifles et

\footnotetext{
${ }^{21}$ Karen Smyley WALLACE, « A search for identity: The Alienated Female Persona in some Francophone African Novels », Rendez-vous: Journal of Arts and Letters, n²2/2, 1986, pp. 32-38, p. 32.

${ }^{22}$ Lylian KESTELOOT, Histoire de la littérature nègre africaine, Karthala, Paris, 2001, p. 284.
} 
de pressions » (CQA, p. 258). Elle s'est heurtée à l'impossibilité de discuter ou de refuser un tel mariage, tandis que son "silence hypothéquait tout [son] avenir $»^{23}$. Arame, déjà liée à un amour réciproque à un jeune homme du village, voit s'effacer devant ses yeux son futur tel qu'elle l'avait imaginé. Elle qui avait envisagé de s'unir à celui qu'elle aimait, doit plutôt partager la couche d'un homme qu'elle déteste : "Frôler cet homme la dégoûtait plus que tout » (CQA, p. 97). Malgré son désir de refuser ce mariage arrangé, elle se plie devant la situation : "À l'époque, elle prenait rendez-vous avec ellemême pour s'écrouler et sangloter, à l'écart de ceux qui, de toute manière, auraient dévalorisé sa détresse en la qualifiant de caprice » (CQA, p. 259). Si son mutisme au moment des faits traduit son impuissance, le roman souligne plutôt une preuve d'humilité.

Non, elle n'était pas faible, en se laissant ainsi écraser par la volonté des autres; elle s'était pliée comme une liane, parce qu'elle ne savait pas comment tiennent les bâtisses sans piliers. Un tuteur, il lui en fallait un, à l'époque et pour toujours, car on l'avait programmé pour la dépendance et la soumission. Son éducation avait toujours été centrée sur son obligation d'alignement aux diktats de la famille, du clan, du village (CQA, p. 258).

De cette vie conditionnée par les décisions prises par d'autres pour son propre destin, il apparaît qu'Arame tire quelques leçons. Lorsque son fils aîné décède tragiquement dans une tempête en mer, il laisse dans le deuil ses femmes et de nombreux enfants qui se retrouvent, dès lors, sans figure masculine. Malgré les pressions des gardiens des traditions, qui commandent dans ces situations un remariage des épouses endeuillées avec un frère du défunt, Arame refuse de piéger son jeune fils Lamine dans la pratique du lévirat : «Lamine était plus jeune que ses belles-sœurs, mais son bref passage à l'école française lui avait ouvert les yeux sur un autre mode de vie [...]. La polygamie, il n'en voulait pas $[\ldots] \gg(C Q A$, p. 15). Toutefois, en d'autres circonstances, Arame n'hésite guère à s'affirmer comme la voix de la perpétuation des traditions. En dépit de sa propre expérience conjugale désastreuse, Arame se plie au raisonnement de de son amie Bougna selon lequel Lamine ne pourrait plus succomber aux charmes l'Occident si une femme l'attendait sur l'île de Niodior. Son amie lui précise les nombreux avantages de l'ajout d'une belle-fille à son précaire équilibre familial : la compagnie d'une autre femme au quotidien, son aide précieuse pour les tâches domestiques, le degré de responsabilités

\footnotetext{
${ }^{23}$ Awa THIAM, La Parole aux négresses, Denoël, Paris, 2001, p. 30.
} 
qui renforcerait le statut d'homme adulte de son seul fils encore vivant. Forte de ces raisonnements et sachant Lamine amoureux de Daba depuis toujours, Arame utilise sans scrupules l'argument d'exilé de son fils pour défaire les fiançailles déjà célébrées de la jeune Daba et son amoureux Ansou.

Très renseignée sur les stratégies matrimoniales de l'île, elles connaissaient la pression que l'entourage était capable d'exercer sur une fille, dès qu'un émigré s'intéressait à elle. Répandre une telle nouvelle, c'était mettre la demoiselle en position de privilégiée, ce qui revenait à lui forcer la main, car tout refus de sa part risquerait de passer pour un caprice (CQA, p. 199).

Le caractère douteux de l'entreprise d'Arame n'a d'égal que la rapidité avec laquelle elle s'empresse de régler l'affaire (et, par le fait même, le sort de Daba). Même son époux Koromâk est incapable de contrecarrer le mariage arrangé entre Daba et Lamine :

- Ce n'est pas très propre tout ça, il y a tout de même d'autres filles à marier dans ce village! On casse la baraque de ce brave Ansou, avec l'assentiment forcé de sa fiancée. Bref, je crois que ce n'est pas ainsi qu' on fait un bon couple.

- Et toi? Comment l'as-tu fait, ton couple? Tu crois que je serais là si mes parents... (CQA, p. 202).

Arame ne manque pas de rappeler à son mari que ses hésitations devant ce mariage forcé auraient été fort pertinentes et opportunes alors qu'il était question de sceller son propre destin. Dans une sorte de revanche du personnage féminin, Arame ne fait que reproduire une pratique couramment exécutée et socialement acceptée, voire encouragée. Il résulte de l'analyse du corps social du roman de Diome que, comme l'exprime Gallimore, « l'État s'est associé à la famille et au clan pour décider du sort de la femme $»^{24}$, sacrifiant sa liberté d'action et d'opinion.

Cette pratique de l'union orchestrée par les pairs est parfois contractée suivant une possibilité d'avancement de la position sociale. Il en découle une équation conjugale où les sentiments et le degré de compatibilité ne sont pas des variables prises en considération. Considérant cela, nous mesurons l'ampleur du veto social tel que présenté dans le roman Celles qui attendent en ce qui concerne les mariages arrangés, et le peu de latitude accordée aux

${ }^{24}$ Béatrice Rangira GALLIMORE, « Le Corps : de l'aliénation à l'appropriation chez les romancières de l'Afrique noire francophone », Notre librairie, 1994, n 117, p. 54-60, p. 57. 
principaux intéressés dans l'éventualité de leur refus. Dans le cas de son fils, Arame se base sur le fait premier des sentiments de Lamine à l'égard de Daba, et sur l'amitié qui les lie, vieille de nombreuses années. Elle applique néanmoins une pression si forte autour de Daba que celle-ci en vient à peser les avantages de la vie de femme mariée à Ansou, un simple pêcheur pour lequel elle éprouve un amour sincère, et ceux de s'unir à Lamine, un homme pour lequel elle n'éprouve que de l'affection, mais qui est auréolé de la gloire de l'exilé. Suivant son désir de valoriser son statut social et se pliant aux pressions ambiantes, Daba emménage dans la demeure d'Arame et commence une vie, à l'image de celle de Coumba, d'épouse jurant fidélité "à sa chambre vide » $(C Q A, 214)$. Étant donné le remous social qui en découlera, Arame ne peut que réaliser l'ampleur de l'erreur qui fût la sienne en poussant Daba à une union avec son fils, alors qu'elle-même a tant souffert de cette coutume.

Arame se sentait mal, parce qu'elle avait tout fait pour marier Lamine et ramener Daba au logis. Avec une assurance qu'elle ne se connaissait pas jusqu'alors, elle avait détruit l'embryon de couple que formaient Ansou et Daba, persuadée que son fils avait mieux à offrir à la jeune fille; et voilà qu'elle en était réduite à la pousser dehors, par manque de ressources. [...] Si Daba devenait aussi malheureuse qu'elle, elle ne se le pardonnerait jamais! (CQA, 226).

Ce n'est que parvenue au terme du roman que le personnage d'Arame se repent de ses actions ; décidant de laisser son fils et sa bru mener leur barque comme ils l'entendent ( $C Q A, 261)$, elle répare en partie l'injure en reconnaissant la marque infligée par ses décisions à la vie de sa belle-fille : "Pardonne-moi d'avoir fait de toi l'épouse d'un absent, car tous tes soucis découlent de là » (CQA, 284-285). Arame incarne donc la figure de la modernité, puisqu'elle met à plat la pratique du lévirat et postule pour une union consensuelle entre son fils et sa bru. En réalité, elle porte et défend les nouvelles valeurs du mariage dans sa communauté.

\section{Conclusion}

En définitive, Celles qui attendent pose la question de l'émancipation des femmes africaines dans des sociétés où les traditions pèsent encore très lourd. En accord avec notre l'analyse, il en ressort qu'Arame, Bougna, Coumba et Daba sont des personnages marginales vivant sous les pressions et les attentes de leur corps social. Le peu de liberté conféré à ce sexe conditionne les héroïnes à une attitude résignation. Imposés aux figures féminines, la sous l'étendard 
des traditions patriarcales, polygamie et le mariage arrangé n'engendrent qu'insatisfaction, misérabilisme et désespoir. Ici, Diome dénonce donc ces « imaginaires crispés » d'une culture obsolète qui se reproduit à l'identique et qui ne peut admettre qu'elle est dépassée. Aussi, l'écrivain ne tombe pas dans le piège d'un féminisme de substitution de l'homme. Cependant, le constat reste mitigé : si certaines femmes comme Arame ont gagné quelques parcelles d'autorité et de liberté, il faut qu'elles accèdent maintenant en plus grande nombre aux formes de mariage qui permettent leur épanouissement.

\section{Bibliographie}

Achola O PALA et Madina LY, La femme africaine dans la société précoloniale, Unesco, Paris, 1979.

Ahmadou KOUROUMA, Les Soleils des Indépendances, Éditions du Seuil, Paris, 1968.

Anne E. BROWN and Marjanne E. GOOZE (Editors), International Women's Writing New Landscapes of Identity, Greenwood Press, Westport, Connecticut, 1995.

Awa THIAM, La Parole aux négresses, Denoël, Paris, 2001.

Béatrice Rangira GALLIMORE, « Le Corps : de l'aliénation à l'appropriation chez les romancières de l'Afrique noire francophone », Notre librairie, 1994, n 117, p. 54-60.

Catherine COQUERY VIDROVITCH, Les Africaines. Histoire des femmes d'Afrique noire du XIXe au XXe siècle, Éditions Desjonquères, Paris, 1994.

Cheik Aliou NDAO, Excellence, vos épouses ! Éditions NEA, Dakar, 1993.

Christiane BERNIER, « Normes et marginalités. Comportements féminins aux $19^{\mathrm{e}}$ et $20^{\mathrm{e}}$ siècles », Recherches féministes, 1992, n $5(1)$, pp. 186-189.

Dominique TABUTIN, «Les populations de l'Afrique au Sud du Sahara », Population et société, 1989, n²31, pp. 40-50.

Gayatri SPIVAK, Les Subalternes peuvent-elles parler? Traduit de l'anglais par Jérôme VIDAL, Éditions Amsterdam, Paris, 2006.

Gayatri SPIVAK, "Perché il pianeta ? Un'autobiografia intellettuale » dans SERGIA Adamo (dirs.), Culture planetarie ? Prospettive e limiti della teoria e della critica culturale. Melmeti. Rome, 2007, p. 41-58.

Irène ASSIBA D'ALMEIDA et Sion HAMOU, « L'écriture féminine en Afrique noire francophone », Études littéraires, vol. 24, Québec, 1991, $\mathrm{n}^{\circ}$, p. $41-50$. 
Karen Smyley WALLACE, «A search for identity: The Alienated Female Persona in some Francophone African Novels », Rendez-vous: Journal of Arts and Letters, 1986, ${ }^{\circ} 22 / 2$, pp. 32-38

Lylian KESTELOOT, Histoire de la littérature nègre africaine, Karthala, Paris, 2001.

Mariama BÂ, Une si longue lettre, Serpent à plumes, Paris, 2001.

Martin T. BESTMAN, Sembene Ousmane et l'esthétique du roman négroafricain, Québec, Naaman, 1981.

Pierrette HERZBERGER-FOFANA, « La Littérature féminine francophone : Les Romancières sénégalaises », Französish heute, vol. 16, 1985, n 4. pp. 407-420.

Sembène OUSMANE, Xala, Présence Africaine, Paris, 1973.

Simone de BEAUVOIR, Le Deuxième sexe, Tome II, Éditions Gallimard, Coll. « Idées », Saint-Amand, 1949. 